bildes, die von Künstlern ihres Faches meist im Haus erfolgten, vereinzelt auch andernorts, z. B. in Nürnberg und Wien.

Einen Genuss für sich bieten die 155 kleinen Nebenkarten, meist Stadtpläne und Umgebungskarten bekannter interessanter Regionen. Bei vielen Länder- und Regionalkarten überrascht zudem die oft recht zutreffende Wiedergabe des Reliefs, teils als Bergzeichnung gesondert vermerkt, und ihre Einfügung in ein feingliedriges Gewässernetz. Der Zeit voraus ist auch die Wiedergabe der Siedlungen nach Einwohnergrößenklassen. Der Stich der Schrift zeigt gewisse Unterschiede der beteiligten Stecher; zum Teil ist sie übertrieben fein, wenn auf $12 \mathrm{~mm} 10$ Zeilen stehen mit Kleinbuchstabenhöhen von 0,4 mm, wie das Blatt Provinz Pommern 1835. Neben Gewässerund Straßennetz enthalten die von Pr. Ltn. Renner gezeichneten Karten neben Schraffen auch noch Waldsignatur. Das führte zu überladenen Kartenbildern, die aber in dieser unglaublichen Feinheit im Stich, hier bei Pommern von C. Eltricht, gediegen umgesetzt worden sind. Bei der Beurteilung der Lesbarkeit solcher Blätter ist aber zu beachten, dass die Reprintreproduktion eine Rasterung notwendig machte, die technisch zur Auflösung feiner Linien in Punktreihen und damit zu Unschärfe führt. Aus nicht ersichtlichen Gründen sind die meisten Blätter sehr spitz reproduziert mit der Folge häufig kranker Linien. Ebenso ist das kräftige zweistufige Originalkolorit extrem verblasst wiedergegeben, worunter der Gesamteindruck der Karten leidet, wie ein Vergleich mit früheren Reproduktionen der gleichen Karten an anderer Stelle zeigt.

Dem Vorteil des Stahlstichs, hohe Auflagen, durchaus bis 30.000 , problemlos drucken zu können, steht als Nachteil gegenüber, dass auf Stahlstichplatten keine Korrekturen ausgeführt werden können; somit veraltet bei Weiterverwendung der Platten allmählich der Inhalt. Für den heutigen Nutzer kann das von Vorteil sein. Die Karten zeigen unverändert die Verhältnisse kurz vor dem deutlich ausgewiesenen Herstellungsjahr, nur selten wurden offensichtlich ältere Originalkarten benutzt. So bietet der Atlas viel detaillierter als ein Geschichtsatlas das Staatenbild der Jahrzehnte nach den Staats- und Grenzfestlegungen des Wiener Kongresses, ihre Verwaltungsgliederung, das Städte- und Straßennetz dieser Zeit.

Die hohen Auflagen der Titelreihe dieser Kartenfolge haben ganz wesentlich dazu beigetragen, geographische Kenntnisse im gesamten deutschsprachigen Raum und weit darüber hinaus in breitere Kreise zu tragen. Der Preis von 2 Groschen sächs. je Karte, also insgesamt 18 Taler für den Atlas, verteilt über 11 Jahre, hat das ermöglicht. Dieser Preis schließt 10 sich über 1 bis 2 Jahre erstreckende Arbeitsschritte und die dazu nötigen Materialien ein: Herstellung und Transport handgeschöpfter Papierbogen aus Rheinhessen nach Hildburghausen, Material und Herstellung von 12 bis $13 \mathrm{~mm}$ starken plan geschliffe-nen Stahlplatten, die danach poliert und grundiert werden mussten. Unabhängig erfolgten Entwurf und Zeichnung der Karten in Originalgröße, danach die Übertragung auf die Platte und der fehlerfreie Stich aller Elemente des Kartenbildes, dann Handpressendruck Fläche ge-gen Fläche und schließlich Handkolorit jedes Druckblattes, danach Komplettieren von Lieferungen für den Versand. Nach Postwegen von oft über 100 und mehr Meilen erreichten die Lieferungen den Empfänger.

Der jetzt so preisgünstig angebotene, 1840 abgeschlossene Reprintatlas ist nicht nur allen an Altkarten interessierten Kollegen, sondern auch all denen zu empfehlen, die sich mit der Geschichte des 19. Jahrhunderts beschäftigen. Ein besseres Hilfsmittel, Reisen, Lebenswege und geschilderte Landschaften im Kartenbild zu begleiten, gibt es nicht.

Werner Stams, Radebeul

\title{
Neuerscheinungen
}

Monmonier, Mark (Ed.);

\section{The History of Cartography, Volume 6 Cartography in the Twentieth Century}

University of Chicago Press 2015, set of 2 volumes, 1960 pages, 805 colour plates, 119 halftones, 242 line drawings, 61 tables, 8-1/2 x 11, cloth 500,00 \$, ISBN: 978-0-226-534695

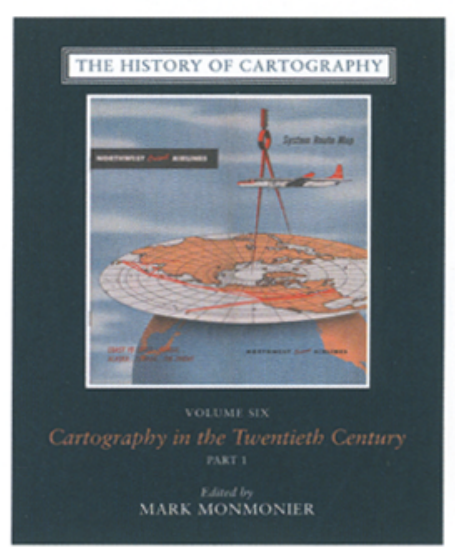

Bryars, Tim; Harper, Tom:

\section{A History of the Twentieth Century in 100 Maps}

224 pages | 100 colour plates

$81 / 2 \times 11 \mid$ (C) 2014

University of Chicago Press 2014

Cloth 45,00 \$,

ISBN: 9780226202471 (for sale in

North and South America only)

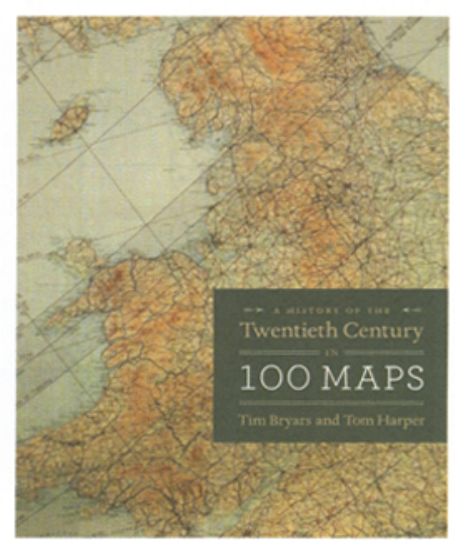

\section{Geoinformation aktuell Business News \\ Mitteilungen aus Wirtschaft und Verwaltung}

\section{Salzburger Know-how im Bereich Geoinformation unterstützt Ärzte ohne Grenzen bei Planung der Hilfsmaßnahmen für Flüchtlinge}

Hoch aufgelöste Satellitenbilder stellen in schwer zugänglichen Gebieten häufig die einzige Informationsquelle zum Aufenthaltsort von Flüchtlingen dar. Um Menschen in Zentral- und Ostafrika helfen zu können, greift Ärzte ohne Grenzen auf Geoinformation von Z_GIS zurück. Informationen zu Anzahl, Aufenthaltsort und Migrationsbewe- gungen der Flüchtlinge ermöglichen rasche Hilfe und effiziente medizinische Vorsorge.

Mit Satellitenbildern und daraus abgeleiteter Geoinformation liefert der Fachbereich Geoinformatik der Universität Salzburg - Z_GIS der Hilfsorganisation Ärzte ohne Grenzen Informationen, die diese bei der Planung und Organisation von Hilfseinsätzen unterstützen. Einen Schwerpunkt bildet dabei Zentralund Ostafrika, insbesondere der Südsudan, wo seit 2013 rund zwei Millionen Menschen auf der Flucht sind. Die 2011 ins Leben gerufene 